\title{
Internal quality assurance activities of a surgical pathology department in an Australian teaching hospital
}

\author{
Ibrahim M Zardawi, Genevieve Bennett, Sanjiv Jain, Michael Brown
}

\begin{abstract}
Aim-To assess the role of a quality assurance programme in improving the service provided by a surgical pathology department.

Methods-A continuous internal quality assurance study of the activities of an anatomical pathology department in an Australian teaching hospital was undertaken over a five year period. This addressed all steps involved in the production of a surgical pathology report. These were addressed in an open forum which included technical, scientific, clerical, and medical staff. Minor errors not needing immediate action were discussed and incorporated into laboratory practice. For major discrepancies with potential implications for patient management supplementary reports were issued and the relevant clinician informed of the outcome.
\end{abstract}

Results-Comprehensive peer review of $8.9 \%$ of the total workload of the department (3530 cases) and all the frozen sections (916 cases) over a period of five years, beginning in 1991, led to comments on some aspects of the original report by the reviewer in $19.6 \%$ of the cases. The great majority of the comments were minor, concerning issues related to the microscopic findings (4\%), macroscopic description (3.1\%), clerical aspects (3\%), typographical errors (3\%), coding errors (2.7\%), technical errors including poor sections and incorrect labelling (1.7\%), inadequate clinical history $(1.2 \%)$, and incomplete or incomprehensible diagrams $(0.9 \%)$. In two cases $(0.05 \%)$ the original report did not state proximity of the tumour to surgical margins and in three of the frozen sections $(0.3 \%)$ the original diagnosis was incorrect. However, in these cases the frozen section assessment did not alter the overall management of the cases. Conclusions-This study highlights the importance of a review system in detecting errors in surgical pathology reporting. Recognition of the fact that surgical pathology is not infallible has improved the end product. It has also minimised interobserver variability in the department, resulting in a uniform approach among the pathologists to macroscopic description, specimen sampling, special stains, and histological reporting. (F Clin Pathol 1998;51:695-699)

Keywords: quality control; audit; performance monitoring; surgical pathology
Surgical pathology should be a gold standard of clinical medicine but it suffers greatly from subjectivity. For this reason, assessment of quality in histopathology and cytopathology is rather difficult. ${ }^{1}$ Because major therapeutic decisions with important implications for patient management are based on histological reports, local audit systems have become necessary. ${ }^{2}$ In an editorial in the Fournal of Pathology, Fleming acknowledged these problems in cellular pathology and asked pathologists to recognise the scale of the problem and adopt an evidence based approach to the issue. $\mathrm{He}$ also advocated other measures, including audit, continuing medical education, and quality assurance schemes. ${ }^{3}$ Our article assesses some of the internal quality assurance and quality control measures and educational programmes that are in place in this laboratory.

\section{Methods}

Anatomical pathology is a section of Australian Capital Territory (ACT) Pathology at The Canberra Hospital, which encompasses surgical pathology, cytopathology, electron microscopy, and postmortem pathology. The Canberra Hospital is a teaching and tertiary referral centre for the Australian Capital Territory and southern New South Wales. One of the clinical schools of The University of Sydney is located on the hospital campus. Computerisation of the laboratory took place in 1987.

An internal quality assurance system for histopathology was devised in 1991 with the aim of conducting a system review, correcting system problems, and adopting a uniform approach to reporting. This is achieved by peer review of approximately $10 \%$ of the surgical pathology workload, and all the frozen sections, on a weekly basis by pathologists not involved in the reporting of the cases under review. The original pathologist, when reporting a routine surgical pathology specimen, is unaware of whether the case will be selected for review. The surgical pathology cases are selected using a systematic sampling method by taking every 10 th number between 0 and 9 . Every case ending with that number, for that particular week, is selected for the review. The meetings are attended by all the pathologists and registrars and by representatives of the scientific and technical staff. Pathologists from the private pathology laboratories in the ACT are invited and a list of cases with weekly laboratory statistics is mailed to them. The previous week's necropsies are also discussed. Any housekeeping matters, including technical issues, are also addressed at the meeting and 
Table 1 Historical account of policies adopted by the laboratory during the period of the study

\begin{tabular}{|c|c|}
\hline July 1991 & How to flag reports needing supplementary report. \\
\hline August 1991 & Laboratory manual to include coding philosophy. \\
\hline September 1991 & Record relevant previous cytology/histology numbers in the report. \\
\hline October 1991 & The "Sydney" classification of gastritis to be adopted. \\
\hline November 1991 & $\begin{array}{l}\text { Future internal QA reviews to include all frozen sections. Presence or absence of transformation zone or squamocolumnar junction to be } \\
\text { mentioned in all cervical biopsies. Gleasons grading system to be used in reporting prostatic adenocarcinomas. }\end{array}$ \\
\hline December 1991 & Key to tissue blocks to be included in the macroscopic description. \\
\hline March 1992 & $\begin{array}{l}\text { Pathologists to review internal QA material on a rotational basis Frozen section diagnosis given to the surgeon to be documented on the reverse } \\
\text { side of the request form, in the surgical pathology report and also in patients' notes. }\end{array}$ \\
\hline April 1992 & Macroscopic description to be changed when further blocks taken. Specimen radiographs to be filed with the report. \\
\hline May 1992 & Reflux oesophagitis to be graded, using a I to IV grading system \\
\hline July 1992 & When asking for recuts, the number of levels already available to be indicated on the recut request sheet for sequential numbering of the levels. \\
\hline December 1992 & Standardise gallbladder sectioning. Pathologist to follow up flow cytometry results before issuing the final report on lymph nodes. \\
\hline February 1993 & $\begin{array}{l}\text { Key to blocks to be specific enough to allow mental reconstruction of the specimen. Standard cutting of radical prostatectomy specimens. Protocol } \\
\text { for handling lumpectomy specimens from mammographically detected abnormalities established. }\end{array}$ \\
\hline March 1993 & Protocol for "tissue audit" specimens introduced. \\
\hline April 1993 & Reviewing pathologist to check, amend and add SNOMED codes when necessary. \\
\hline July 1993 & "Q probe" study on orientated skin specimens by one of the pathologists. Note in report if photography performed on the macroscopic specimen. \\
\hline August 1993 & "Q probe" study on liver biopsies by one of the pathologists. \\
\hline September 1993 & Record in frozen section report the number of nodes found and sectioned. \\
\hline November 1993 & Significance of atypical ductal hyperplasia in breast cores with regards to further management. \\
\hline February 1994 & Reflux oesophagitis grading revisited. \\
\hline May 1994 & Quantification of helicobacter-like organisms in gastric biopsies \\
\hline July 1994 & Scheuer's grading system of chronic hepatitis to be adopted. \\
\hline December 1994 & Referred cases to be recut and original slides kept in the laboratory. \\
\hline January 1995 & Discussion on grading of dysplasia (low grade and high grade versus mild, moderate, and severe). \\
\hline May 1995 & Reports on breast cores to include comments on correlation with mammographic findings. \\
\hline May 1996 & Discussion on dysplastic naevi versus melanoma in situ. Block for resection margins on oesophagectomy specimens to be circumferential. \\
\hline June 1996 & Strict criteria for dysplastic naevi to be used. \\
\hline July 1996 & Standardised reporting of gastritis to be adopted. \\
\hline September 1996 & Need to indicate number of levels cut already on recuts requested. Pathologists still not uniformly reporting gastritis. \\
\hline February 1997 & Pathologists to specify the grading system used in the report. Reintroduce review of weekly necropsies. \\
\hline March 1997 & Use of standard reporting formats for some tumours. \\
\hline
\end{tabular}

changes to the current practices are discussed and documented in the pathology manual. An overview and summary of any recent scientific meetings attended by staff, and quality assurance cases of the Royal College of Pathologists of Australasia, are also presented at these meetings.

The review process examines all aspects of the case including patient demographics, typing errors, coding, adequacy of clinical history, technical quality and labelling of the histological slides, diagrams, macroscopic description, key to blocks, microscopic reports, and minor and major discordant diagnoses. Turn around time (time from specimen collection to report completion), adequacy of specimen sampling, and use of special stains are also assessed. Records are kept for each meeting and a semi-

Table 2 Frequency of comment in each category examined, from a total sample of 3530 cases

\begin{tabular}{lc}
\hline Variable & $\begin{array}{c}\text { Number and percentage } \\
\text { of cases with comments }\end{array}$ \\
\hline History & $42(1.2 \%)$ \\
Typographical & $107(3.0 \%)$ \\
Clerical & $109(3.0 \%)$ \\
Technical & $61(1.7 \%)$ \\
Diagrams & $31(0.9 \%)$ \\
Macroscopic & $110(3.1 \%)$ \\
Microscopic & $142(4.0 \%)$ \\
Diagnosis & $2(0.05 \%)$ \\
Coding & $97(2.7 \%)$ \\
Total & $706(19.6 \%)$ \\
\hline
\end{tabular}

quantitative system for documenting errors is used. Discrepancies are generally graded as minor-not requiring a supplementary report-and major, with implications for patient management necessitating a supplementary report.

\section{Results}

Table 1 is a historical account of the laboratory system policies in histopathology adopted as a result of discussions in these quality assurance meetings since 1991, in chronological order.

Of 39476 surgical pathology specimens received in the department during the period of the study (225 working weeks), 3530 cases $(8.9 \%)$ were actually reviewed. In $19.6 \%$ of these, the reviewer commented on aspects of the cases under review. Table 2 shows the frequency and percentage of comments for each of the variables under investigation.

Table 3 shows the frequency and percentage of comments for each of the variables. Complete data was available for the period 1992 to 1996 . Comments on the microscopic opinions were the commonest at $18 \%$, followed by comments on the macroscopic description (16\%), clerical aspects (15\%), typographical $(15 \%)$, coding (14\%), technical (9\%), clinical history $(6 \%)$, diagrams $(4 \%)$, and diagnosis $(1 \%)$. The great majority of the histological discrepancies were minor and included issues

Table 3 Percentages of observations for each variable assessed for 1992 to 1996

\begin{tabular}{lrrllllllll}
\hline Year & History & \multicolumn{1}{l}{ Typo } & Clerical & Technical & \multicolumn{1}{c}{ Diagrams } & Macro & \multicolumn{1}{l}{ Micro } & Dx/Fs & Code & Total \\
\hline 1992 & $10(8 \%)$ & $9(7 \%)$ & $16(13 \%)$ & $11(9 \%)$ & $10(8 \%)$ & $15(12 \%)$ & $29(23 \%)$ & $1(1 \%)$ & $26(19 \%)$ & 127 \\
1993 & $14(9 \%)$ & $19(12 \%)$ & $14(9 \%)$ & $19(12 \%)$ & $7(4 \%)$ & $28(18 \%)$ & $32(20 \%)$ & $2(1 \%)$ & $23(15 \%)$ & 158 \\
1994 & $12(8 \%)$ & $22(14 \%)$ & $34(21 \%)$ & $15(9 \%)$ & $5(3 \%)$ & $18(11 \%)$ & $31(20 \%)$ & $2(1 \%)$ & $21(13 \%)$ & 160 \\
1995 & $4(2 \%)$ & $32(20 \%)$ & $31(17 \%)$ & $13(6 \%)$ & $5(5 \%)$ & $30(13 \%)$ & $23(19 \%)$ & $1(1 \%)$ & $20(12 \%)$ & 159 \\
1996 & $2(8 \%)$ & $25(12 \%)$ & $14(11 \%)$ & $3(15 \%)$ & $4(4 \%)$ & $19(13 \%)$ & $27(17 \%)$ & $1(1 \%)$ & $7(19 \%)$ & 102 \\
Total & $42(6 \%)$ & $107(15 \%)$ & $109(15 \%)$ & $61(9 \%)$ & $31(4 \%)$ & $110(16 \%)$ & $142(20 \%)$ & $7(1 \%)$ & $97(14 \%)$ & 706 \\
\hline
\end{tabular}

Typo, typographical; macro, macroscopic; micro, microscopic; Dx, diagnosis; Fs, frozen section. 
Table 4 Frequency of frozen sections in different anatomical sites

\begin{tabular}{|c|c|c|c|c|c|c|c|c|c|c|c|}
\hline Year & $L N$ & Brain & $G I T$ & Breast & Thorax & Thyroid & P/Thyroid & Oral & Renal & Misc & Total \\
\hline 1992 & $14(10 \%)$ & $34(25 \%)$ & $19(14 \%)$ & $26(19 \%)$ & $9(7 \%)$ & $14(10 \%)$ & $4(3 \%)$ & $6(4 \%)$ & $4(3 \%)$ & $7(5 \%)$ & 137 \\
\hline 1993 & $29(19 \%)$ & $46(29 \%)$ & $13(8 \%)$ & $23(15 \%)$ & $8(5 \%)$ & $14(9 \%)$ & $3(2 \%)$ & $5(3 \%)$ & $1(1 \%)$ & $15(9 \%)$ & 157 \\
\hline 1994 & $19(10 \%)$ & $44(23 \%)$ & $24(12 \%)$ & $31(16 \%)$ & $19(10 \%)$ & $19(10 \%)$ & $2(1 \%)$ & $8(4 \%)$ & $4(2 \%)$ & $23(12 \%)$ & 193 \\
\hline 1995 & $46(23 \%)$ & $41(20 \%)$ & $17(9 \%)$ & $32(16 \%)$ & $10(5 \%)$ & $16(8 \%)$ & $5(3 \%)$ & $9(5 \%)$ & $2(1 \%)$ & $20(10 \%)$ & 198 \\
\hline 1996 & $53(23 \%)$ & $46(20 \%)$ & $21(9 \%)$ & $32(14 \%)$ & $12(5 \%)$ & $18(8 \%)$ & $16(7 \%)$ & $12(5 \%)$ & $2(1 \%)$ & $19(8 \%)$ & 231 \\
\hline Total & $161(18 \%)$ & $211(23 \%)$ & $94(10 \%)$ & $144(16 \%)$ & $58(6 \%)$ & $81(9 \%)$ & $30(3 \%)$ & $40(4 \%)$ & $13(2 \%)$ & $84(9 \%)$ & 916 \\
\hline
\end{tabular}

LN, lymph node; GIT, gastrointestinal tract; P/thyroid, parathyroid; Misc, miscellaneous.

such as grading of various pathological processes, which suffers from subjectivity.

In the same period all the 916 frozen sections examined were also reviewed. Detailed analysis of the frozen sections for the complete five year period is shown in table 4 . Frozen sections are performed on almost all the intracranial lesions operated on, which accounted for $23 \%$ of all the frozen sections in the laboratory. Pelvic lymph nodes $(18 \%)$ from patients with prostatic or vesical malignancies have increased in recent years. Frozen sections from breast and gastrointestinal tract $(16 \%$ and $10 \%$ respectively) have changed little, as have those from thoracopulmonary sites $(6 \%)$, thyroid $(9 \%)$, parathyroid (3\%), mouth $(4 \%)$, and kidney (2\%).

Details of the five discordant frozen sections results are shown in table 5 . None of the revised diagnoses altered the immediate management plans for the patients involved. The testicular embryonal carcinoma had a sprinkling of lymphocytes which made the pathologist suggest seminoma. The two cases of metastatic malignancy in regional lymph nodes included metastatic melanoma in a groin lymph node and micrometastasis from a well differentiated prostatic adenocarcinoma in a pelvic lymph node. The metastatic melanoma was diagnosable on the actual frozen section but the metastatic adenocarcinoma was only seen on the paraffin embedded tissue from the frozen section sample. The peer review of the intracranial frozen sections highlighted two cases. A case of schwannoma which had been called meningioma and a case of low grade astrocytoma which was not apparent on the brain smears or the frozen section were identified. The neoplastic tissue was only seen in the paraffin block of the frozen section. In none of the cases did the revised diagnosis change the clinical management.

The final histological diagnosis was revised in two cases. The tumour in each case was abutting surgical margins, which had not been mentioned in the report. In view of the high grade of the breast cancer one patient received radiotherapy; a computer search failed to show

Table 5 The discordant frozen section

\begin{tabular}{lll}
\hline FS diagnosis & Review diagnosis & Paraffin diagnosis \\
\hline Seminoma & Embryonal carcinoma & Embryonal carcinoma \\
No definite tumour & Metastatic melanoma & Metastatic melanoma \\
Meningioma & Schwannoma & Schwannoma \\
Normal brain tissue & Normal brain tissue & Low grade astrocytoma \\
Reactive lymph node & Reactive lymph node & Metastatic adenocarcinoma \\
\hline
\end{tabular}

FS, frozen section. follow up information on the other patient, who had prostatic carcinoma.

\section{Discussion}

Quality is defined as the sum total of features and characteristics of a service that bear on its ability to satisfy the user's needs. Quality assurance is a management system designed to achieve an acceptable level of quality service which satisfies the user's requirements with minimum expenditure. Quality control involves the operational techniques and activities that are used to fulfil specified requirements for quality, which means checking each item before it is issued and discarding those that fail to meet approved quality standards. ${ }^{14}$ Quality system is the framework of the organisational structure and includes standardised procedures which support the operation of an enterprise. In the laboratory environment, quality control is the day to day monitoring of reproducibility, designed to detect any serious errors in the day's procedures. ${ }^{5}$

Several essential facets of product quality have been identified by the International Standards Organisation (ISO). ${ }^{6}$ These include defining and updating a product, designing into the product certain characteristics, maintaining day to day consistency of the product, furnishing support through the life cycle of the product that enables it to meet marketplace requirements and opportunities, and providing value for the customer. These recommendations have certain implications for the observer dependent field of surgical pathology. Morphological features used in categorising lesions should be reproducible, and objective criteria should be used to assess various histological and cytological variables. There are good data on external quality assurance schemes in histopathology and cytopathology, but there is a lack of published information on internal audits. ${ }^{7-11}$ In a prospective study of 3000 surgical pathology cases, Whitehead et al identified disagreement between the preliminary original diagnosis and the reviewer's assessment which led to modification of the final diagnosis in $7.8 \%$, and in $0.96 \%$ of the cases the changes in the diagnosis were significant. ${ }^{8}$ In our study the overall microscopic discrepancies between the original description and the review was $4 \%(142 / 3530)$ and the final diagnosis was altered in $0.05 \%$ of surgical pathology cases (7/3530) and in $0.3 \%$ of the frozen sections (three cases).

In auditing diagnostic performance during clinicopathological meetings, McBroom and Ramsay reviewed 416 cases at 58 meetings over a period of 14 weeks. ${ }^{12}$ The histological review 
resulted in an altered diagnosis in $9 \%$, a refined diagnosis in $10 \%$, and no diagnostic change in $81 \%$ of the cases. In approximately $90 \%$ of the cases the changes were attributed to the specialist expertise of the reviewing pathologist, and in about $5 \%$ the change in the diagnosis resulted from the availability of additional clinical information. Amended diagnoses led to major management changes in $3.8 \%$ and to minor changes in $2.9 \%$ of the cases. Although the method of selection of cases for review in this article provided valuable information on departmental diagnostic performance, it did not lend itself to a formal systematic audit scheme. Our system is more comprehensive than the model used by McBroom and Ramsay because it selects cases at random and addresses all aspects involved in the generation of anatomical pathology reports. It creates a subconscious awareness of the requirements of the department, assists in standardising the approach to reporting, and leads to a fall in error rates. The overall observations made by the reviewers did not decline since during the period of the study the workload almost doubled. Other factors include staff changeover at all levels, the non-parametric nature of the discipline, and the subjectivity of the pathologists' comments. We therefore agree with Ramsay ${ }^{2}$ that "closing the audit circle" requires measurement of improvement and we have been able to demonstrate this in our study.

In a study on the accuracy of frozen section diagnosis from the Mayo Clinic Rochester, Ferreiro et al analysed retrospectively the results of 97914 frozen section slides from 24880 cases and found an accuracy rate of $97.8 \%$, with $2.2 \%$ of the results requiring revision $(1.6 \%$ unavoidable due to tissue sampling, $0.5 \%$ reflecting change in the degree of abnormality, and $0.1 \%$ clinically significant errors). ${ }^{13}$ Our data on frozen sections showed $0.3 \%$ discordance with the review diagnosis (3/916). The review findings did not adversely affect the patients concerned.

Our work and other studies have shown that anatomical pathology reports are not infallible. ${ }^{512}$ Pathologists should endeavour to minimise their reporting errors, not just for the diagnostically difficult cases but across the whole spectrum of daily reporting. Each anatomical pathology laboratory should therefore establish and maintain a system appropriate to the type, range, and volume of tests it undertakes. The laboratory should define and document its policies and objectives for good practice. Clearly defined procedures should be available for feedback and corrective action whenever discrepancies are detected or departures from documented polices occur. To continue operating within the requirements of the quality system, the laboratory should audit its activities at appropriate intervals. These audits should be carried out by trained and qualified staff who are independent of the activity that is being assessed. All audit and review findings should be documented, and immediate action should be implemented and the "client" informed in writing if the findings cast doubt on the correctness or validity of a test result. ${ }^{14}$ In our study the findings of the audit were used as an internal educational tool to improve reporting of surgical pathology specimens and to achieve uniformity of reporting. In addition, the clinicians concerned were notified of any discrepant results that required a supplementary report.

Although intraobserver variability is less than interobserver variability, the problem of subjectivity, which has always been with us, is coming increasingly under scrutiny. As management decisions are being made on features that are not consistently reproducible, it is important that we, as a professional body, address this issue, since the remit of health care providers is to improve the effectiveness of patient care with ever diminishing health resources. ${ }^{15-17}$ In a study by Safrin and Bark, ${ }^{18}$ the value of a dual pathologist signout was investigated. A second pathologist routinely reviewed all the 5397 surgical pathology cases for the one year duration of the study and found an error rate of $0.26 \%$ at a cost of US $\$ 7$ per case or US $\$ 2700$ for each discrepancy of potential clinical significance. Our study did not explore the cost of the activities that are undertaken in our department but the information it provides goes beyond spotting diagnostic errors by examining all aspects of the practice of anatomical pathology. To minimise interobserver and intraobserver variability, each anatomical pathology department should institute measures that will remedy this shortcoming adequately. These may include internal audits, participation in external quality assurance schemes, clinicopathological correlations, good communication and exchange of ideas with clinicians, follow up of unusual and interesting cases, and continuing professional education. ${ }^{14}$

In a recent questionnaire based survey of errors in diagnostic histopathology throughout the United Kingdom, Furness and Lauder suggest that a typical pathologist probably becomes aware of having made a serious error approximately once a year. ${ }^{19}$ They recommend a larger and more systematic study of the circumstances under which errors are likely to occur. We have undertaken such a systematic study in a continuous way, using internal resources. On average, each of the pathologists devotes one hour per week to matters related to internal surgical pathology quality assurance activities. This includes peer review and attendance at our internal quality assurance meetings. In addition one of the registrars who selects the cases and assigns them to the reviewers spends approximately 30 minutes a week on this aspect of the activity. It takes clerical staff 60 minutes a week to find the slides and the reports for the reviewing pathologist. The time spent by the pathologists, registrars, and the technical and scientific staff attending the weekly meeting is also taken into consideration when calculating the cost of the exercise. The overall cost of the activity per year is approximately AUS $\$ 18000.00$, which equates to AUS $\$ 1.50$ for each surgical pathology case processed in the laboratory.

Our five year experience with the internal quality assurance activities described here has 
provided the department with the following benefits:

(1) Since any error may be picked up in the review process, there is a stimulus at both subconscious and conscious level to be more accurate.

(2) There is more frequent case consultation among the pathologists.

(3) Uniformity in diagnostic terminology, grading systems, and criteria across all the pathologists has been achieved.

(4) Team work mentality has also been achieved, since pathologists work together rather than individually for the benefit of the department.

(5) There is continuous education and exchange of ideas, concepts, and information among all involved.

(6) Collective decision making regarding procedures, practices, and quality increases the chance of compliance with such decisions.

(7) Feedback to the technical and scientific staff in terms of the technical quality and efficiency of the department is more successful in a group situation than individually.

(8) Our quality assurance activities have created a good working environment with common goals.

CONCLUSIONS

In summary, the most important outcome of such a quality assurance exercise in this laboratory has been the opportunity to bring all members of the laboratory together at regular intervals to discuss the scientific and technical aspect of the operations of the team. Such a forum has provided an avenue for exchange of ideas and has offered every person in the laboratory the opportunity to contribute positively to the activities of the department. This has been an effective way of remedying any problem. It has also assisted in providing a uniform approach to most issues, has discouraged building systems around individuals, and has made staffing the department at all times relatively easy. In order to produce consistent high quality reports on anatomical pathology specimens, ongoing involvement in these activities by all members of the department is essential. Finally, we agree with Gibbs and Kirkham ${ }^{20}$ that an audit is not an end in itself and that laboratories need to recognise trends that can lead to improvements in performance.

We acknowledge the assistance of many of the registrars and the scientific and technical staff of anatomical pathology at The Canberra Hospital.

1 Codling BW. Audit, quality assurance and quality control in histopathology. In: Anthony PP, MacSween RNM, eds. Recent advances in histopathology,

2 Ramsay AD. Locally organised medical audit in histopathology. F Clin Pathol 1991;44:353-7.

3 Fleming KA. Evidence-based pathology. F Pathol 1996;179: $127-8$.

4 Australian Department of Administrative Services. Definitions in quality assurance policy. Canberra: Australian Government Publishing Service, 1992:3-7.

5 Kilshaw D. Quality assurance 2. Internal quality control. Med Lab Sci 1987;44:73-83.

6 Australian/New Zealand Standard (ISO 9002). Quality systems model for quality assurance in production, installation and servicing. Canberra: Standard Australia, 1994:1-21.

7 Zuck JA, Kenyon WE, Myskow MW. Audit in histopathology: description of an internal quality assessment scheme with analysis of preliminary results. $\mathcal{f}$ Clin Pathol 1991;44:10-16.

8 Whitehead ME, Fitzwater JE, Lindley SK, et al. Quality assurance of histopathologic diagnosis: a prospective audit of three thousand cases. Am f Clin Pathol 1984;81:487-91.

9 O'Sullivan JP, Ismail SM, Barnes WS, et al. Interobserver variation in the diagnosis and grading of dyskaryosis in cervical smears: specialist cytopathologist compared with non-specialists. $\mathcal{F}$ Clin Pathol 1994;47:515-18.

10 Zarbo RJ, Howanitz PJ, Bachner P. Interinstitutional comparison of performance in breast fine needle aspiration cytology. A Q-probe quality indicator study. Arch Pathol Lab Med 1991;115:743-50.

11 Zarbo RJ, Hoffman GG, Howanitz PJ. Interinstitutional comparison of frozen section consultation. A college of American Pathologists Q-probe study of 79647 consultations in 297 North America institutions. Arch Pathol Lab Med 1992;116:1187-94.

12 McBroom HM, Ramsay AD. The clinicopathological meeting. A means of auditing diagnostic performance. $A m \mathcal{F}$ ing. A means of auditing
Surg Pathol 1993;1 17:75-80.

13 Ferreiro JA, Myers JL, Bostwick DG. Accuracy of frozen section diagnosis in surgical pathology: review of a 1 year experience with 24880 cases at Mayo Clinic Rochester. Mayo Clin Proc 1995;70:1137-41.

14 Bonfiglio TA, Corson JM, Fechner RE, et al. Recommendations on quality control and quality assurance in anatomic pathology. Association of directors of anatomic and surgical pathology. Am f Surg Pathol 1991;15:1007-9.

15 McCluggage WG, Bharucha $\mathrm{H}$, Caughley LM, et al. Interobserver variation in the reporting of cervical colposcopic biopsy specimens: comparison of grading systems. $\mathcal{F}$ Clin Pathol 1996;49:833-5.

16 Woodhouse SL, Wagner E. Managed discontinuity of care: the value and fate of cytohistologic correlation. Diagn Cytopathol 1997;16:105-6.

17 Zarbo RJ. Quality assurance in anatomic pathology in the cost-conscious era. Am f Clin Pathol 1996;106(suppl 1):3-

18 Safrin RE, Bark CJ. Surgical pathology sign-out. Routine review of every case by a second pathologist. Am F Surg Pathol 1993;17:1190-2.

19 Furness PN, Lauder I. A questionnaire-based survey of errors in diagnostic histopathology throughout the United Kingdom. $\mathcal{f}$ Clin Pathol 1997;50:457-60.

20 Gibbs N, Kirkham N. Clinical audit in histopathology. In: Kirkham N, Hall P. Progress in pathology, vol 1. Edinburgh: Churchill Livingstone, 1995:213-28. 